\title{
FATORES ASSOCIADOS À PRESSÃO ARTERIAL INADEQUADA DE PESSOAS COM HIPERTENSÃO*
}

\author{
Anderson da Silva Rêgo ${ }^{1}$, Victória dos Santos Laqui², Fernanda Gatez Trevisan², André Estevam Jaques ${ }^{3}$, \\ Rosana Rosseto de Oliveira ${ }^{4}$, Cremilde Aparecida Trindade Radovanovic ${ }^{5}$
}

\begin{abstract}
RESUMO: Objetivo: avaliar os fatores associados à pressão arterial inadequada em pessoas com hipertensão acompanhadas pela Estratégia Saúde da Família. Método: estudo transversal, realizado com 417 pessoas, no município de Maringá, Paraná. A coleta de dados foi realizada no primeiro semestre de 2016, por meio do instrumento satisfação do usuário com hipertensão arterial com os serviços prestados pela Atenção Primária à Saúde, utilizando as questões referentes ao perfil sociodemográfico, nutricional e clínico. Na análise dos dados, aplicou-se estatística descritiva e inferencial. Resultados: a maioria dos entrevistados, $62,4 \%$ era idoso, $67,8 \%$ do sexo feminino e 55,2\% aposentado/pensionista. Houve associação da pressão arterial inadequada com pessoas de idade superior a 59 anos, aposentados/pensionistas, moderadamente ativos, não praticantes de atividades físicas e fragilidade nas orientações sobre a doença. Conclusão: os resultados sinalizam a necessidade de reorganização do processo de trabalho, com o fortalecimento das orientações e educação em saúde às pessoas com hipertensão arterial.
\end{abstract}

DESCRITORES: Hipertensão; Fatores de risco; Prevenção de doenças; Estratégia Saúde da Família; Enfermagem.

\section{FACTORS ASSOCIATED WITH INAPPROPRIATE BLOOD PRESSURE IN HYPERTENSIVE PATIENTS}

ABSTRACT: Objective: assess the factors associated with inappropriate blood pressure in hypertensive patients monitored in the Family Health Strategy. Method: cross-sectional study, involving 417 people in Maringá, Paraná. The data were collected in the first semester of 2016, using the instrument satisfaction of hypertensive users with Primary Health Care services; using the questions related to the sociodemographic, nutritional and clinical profile. In the data analysis, descriptive and inferential statistics were applied. Results: most interviewees, $62.4 \%$ were elderly, $67.8 \%$ female and $55.2 \%$ retired/pensioner. Inappropriate blood pressure was associated with people over 59 years of age, retired/pensioners, moderately active, without practicing physical exercise and weakly oriented about the disease. Conclusion: the results signal the need to reorganize the work process, strengthening the orientations and health education for arterial hypertension patients.

DESCRIPTORS: Hypertension; Risk factors; Disease prevention; Family Health Strategy; Nursing.

\section{FACTORES ASOCIADOS A LA PRESIÓN ARTERIAL INADECUADA DE PERSONAS CON HIPERTENSIÓN}

RESUMEN: Objetivo: El propósito del estudio fue evaluar los factores asociados a la presión arterial inadecuada en personas con hipertensión acompañadas por la Estrategia Salud de la Familia. Método: Fue un estudio trasversal, realizado con 417 personas, en el municipio de Maringá, Paraná. La obtención de datos ocurrió en el primer semestre de 2016, por medio del instrumento de satisfacción del usuario con hipertensión arterial con los servicios prestados por la Atención Primaria a la Salud, utilizando las cuestiones referentes a los perfiles socio demográfico, nutricional y clínico. En el análisis de los datos, se aplicó estadística descriptiva e inferencial. Resultados: La mayoría de los entrevistados, $62,4 \%$, era de ancianos, $67,8 \%$ del sexo femenino y $55,2 \%$ jubilado/pensionado. Hubo asociación de la presión arterial inadecuada con edades superiores a 59 años, jubilados/pensionados, moderadamente activos, no practicantes de actividades físicas y la debilidad de orientaciones acerca de la enfermedad. Conclusión: Se concluye que hay la necesidad de reorganizar el proceso de trabajo y perfeccionar las orientaciones y la educación en salud de las personas con hipertensión arterial.

DESCRIPTORES: Hipertensión; Factores de riesgo; Prevención de enfermedades; Estrategia Salud de la Familia; Enfermería.

\footnotetext{
*Artigo extraído da dissertação intitulada: "Avaliação da satisfação de pessoas com hipertensão arterial com os serviços prestados pela Atenção Primária à Saúde". Universidade Estadual de Maringá, 2017.

${ }^{1}$ Enfermeiro. Doutorando em Enfermagem. Universidade Estadual de Maringá. Maringá, PR, Brasil.

${ }^{2}$ Discente de Enfermagem. Universidade Estadual de Maringá. Maringá, PR, Brasil.

${ }^{3}$ Enfermeiro. Doutor em Enfermagem. Docente de Enfermagem da Universidade Estadual de Maringá. Maringá, PR, Brasil.

${ }^{4}$ Enfermeira. Pós-doutoranda em Enfermagem. Universidade Estadual de Maringá. Maringá, PR, Brasil.

${ }^{5}$ Enfermeira. Doutora em Ciências da Saúde. Docente de Enfermagem e do Programa de Pós-Graduação em Enfermagem da Universidade Estadual de Maringá. Maringá, PR, Brasil.
}

E-mail: andersondsre@gmail.com 


\section{INTRODUÇÃO}

A hipertensão arterial (HA) é um grande problema de saúde pública mundial ${ }^{(1)}$. Com alta prevalência, é responsável por elevado número de óbitos decorrentes da sua cronicidade, além de demandar assistência multiprofissional, considerando sua origem multifatorial, complexidade e diversidade de sintomas tensionais ${ }^{(2)}$.

A prevalência mundial da HA é de aproximadamente $31 \%$, sendo responsável por $63 \%$ de um total de 38 milhões de óbitos de adultos no mundo. De acordo com a Organização Mundial da Saúde (OMS), o continente Africano possui a maior prevalência de pessoas acima de 25 anos de idade diagnosticadas com HA $(46 \%)$, em contraste com o continente norte-americano, que apresenta menor prevalência $(35 \%)^{(1,3)}$.

No Brasil, a prevalência da doença é aproximadamente $24,8 \%$ em indivíduos adultos, com maior proporção entre as mulheres $(26,8 \%)$ em relação aos homens $(22,5 \%)^{(4)}$. A ocorrência da doença também é maior em indivíduos com baixa escolaridade, o que potencializa a dificuldade em manter os valores pressóricos dentro da normalidade, contribuindo no surgimento de complicações decorrentes de sua cronicidade $^{(4)}$.

Nesse aspecto, o difícil controle pressórico tem levantado a necessidade de estratégias substanciais relacionadas à assistência prestada, sobre o enfrentamento e convivência com a doença ${ }^{(5)}$. A diversidade de fatores que interferem no tratamento e controle da HA, como determinantes comportamentais, estado nutricional, sociodemográfico e de adesão à terapêutica farmacológica, deve ser considerada na criação e adoção de novas estratégias, com avaliação periódica das pessoas pelos profissionais de saúde ${ }^{(5-6)}$.

Nesse sentido, conhecer os fatores associados à pressão arterial inadequada pode favorecer a discussão e aprimoramento da organização do processo de trabalho assistencial, pautados na necessidade de saúde da população, com ações precisas, efetivas e resolutivas ${ }^{(2)}$. Diante do exposto, o objetivo deste estudo foi avaliar os fatores associados à pressão arterial inadequada em pessoas com hipertensão acompanhadas pela Estratégia Saúde da Família (ESF).

\section{MÉTODO}

Trata-se de um estudo de corte transversal, realizado com pessoas em tratamento da HA e acompanhadas pela ESF. O município em que a pesquisa foi realizada localiza-se no noroeste do estado do Paraná, Brasil, possui população estimada de 403.063 habitantes, com sistema primário de saúde organizado de forma descentralizada, composta por 34 Unidades Básicas de Saúde (UBS) e 74 equipes da ESF, perfazendo a cobertura de $68,01 \%$ da população ${ }^{(7-8)}$.

Para o cálculo amostral foram considerados 27.741 indivíduos com HA, cadastrados na rede ambulatorial do Sistema Único de Saúde (SUS) - HIPERDIA. Os parâmetros utilizados para o cálculo de amostragem aleatória simples foram o erro de estimativa de 5\%, 95\% de intervalo de confiança e acréscimo de 15\% para possíveis perdas, totalizando 437 pessoas, posteriormente estratificadas de acordo com o número de pessoas acompanhadas por cada UBS do município. Considerando as perdas e recusas, a amostra final do estudo foi de 417 pessoas.

Foram definidos como critérios de inclusão ter idade igual ou superior a 18 anos, residir na área urbana do município, ter sido atendido por profissionais de saúde das UBS nos últimos seis meses antes da coleta de dados, e possuir cadastro no HIPERDIA realizado até o ano de 2014, para que a análise dos prontuários e do acompanhamento do ano de 2015 pudesse ser realizada. Como critério de exclusão estar gestante no momento da entrevista.

A coleta de dados foi realizada entre os meses de fevereiro e junho de 2016 durante o horário das reuniões do HIPERDIA, que acontecia frequentemente entre as 08:00 horas e as 17:00 horas. Realizouse o contato inicial com os gestores, médicos e enfermeiros das UBS e posteriormente com os sujeitos de pesquisa, sendo apresentado o estudo e realizado o convite para participação. Todas as entrevistas individuais foram realizadas em ambientes confortáveis e livre de interferência. 
Foram utilizados dois instrumentos para coleta de dados. O primeiro avaliou e classificou a população quanto ao nível econômico ${ }^{(9)}$. O segundo avaliou a satisfação do usuário com HA com os serviços prestados pela Atenção Primária à Saúde (APS), adaptado e validado por Paes ${ }^{(10)}$. Para este estudo, utilizou-se as variáveis do primeiro bloco de questões referentes ao perfil sociodemográfico, nutricional e clínico dos entrevistados.

Utilizaram-se equipamentos para aferição dos dados antropométricos e valores pressóricos, com regulagem e calibração atuais, considerando as diretrizes da Sociedade Brasileira de Cardiologia (SBC) para as técnicas de coleta das informações referidas ${ }^{(11)}$. Utilizou-se balança analógica com régua antropométrica para verificar peso e altura e esfigmomanômetros do tipo aneroide para aferição da pressão arterial. Para verificação da circunferência abdominal, foi utilizada fita inelástica de 150 centímetros.

Os valores de pressão arterial foram categorizados em Controle Pressórico Adequando quando a pressão arterial sistólica (PAS) era $\leq 140 \mathrm{mmHg}$ e pressão arterial diastólica (PAD) $\leq 90 \mathrm{mmHg}$, conforme os critérios da VII Diretriz Brasileira de Hipertensão Arterial ${ }^{(11)}$.

O acompanhamento regular dos usuários foi estabelecido de acordo com os dados referentes ao ano de 2015 como base para avaliação, seguindo os critérios propostos pelo Ministério da Saúde ${ }^{(12-13)}$, que considera acompanhamento regular o comparecimento à UBS por no mínimo três vezes ao ano, com valores pressóricos aferidos e registrados no prontuário.

Para a circunferência abdominal (CA) e o índice de massa corpórea (IMC), foi adotado o ponto de corte estabelecido pelas diretrizes da Associação Brasileira para o Estudo da Obesidade e Síndrome Metabólica $(\mathrm{ABESO})^{(14)}$, que considera CA alterada quando os valores são $\geq 94$ centímetros para homens $\mathrm{e} \geq 80$ para as mulheres. O IMC foi calculado e categorizado em baixo peso (IMC $<18,5 \mathrm{~kg} / \mathrm{m}^{2}$ ), eutrófico (IMC entre 18,5 e 24,9 kg/m²), sobrepeso (IMC entre 25 e $29,9 \mathrm{~kg} / \mathrm{m}^{2}$ ) e obesidade grau I (IMC entre 30 a $\left.34,9 \mathrm{~kg} / \mathrm{m}^{2}\right)$, grau II (IMC entre 35 e $\left.39,9 \mathrm{~kg} / \mathrm{m}^{2}\right)$ e grau III $\left(\text { IMC }>40 \mathrm{~kg} / \mathrm{m}^{2}\right)^{(14)}$.

Para as variáveis referentes às complicações da HA, tais como possuir diabetes Mellitus (DM) tipo um ou dois e o número de medicações em uso para tratamento da morbidade, consideraram-se as informações autorreferidas pelos entrevistados e posteriormente foram confirmadas na análise dos prontuários. Para a variável tabagismo, os entrevistados foram questionados quanto ao consumo ativo de cigarros, de acordo com os critérios recomendados pelo Ministério da Saúde, que considera tabagista aquele com consumo de no mínimo cinco cigarros por dia ${ }^{(15)}$.

Para a classificação quanto à realização de exercícios físicos regulamente, foi considerado ativo o usuário que relatou realizar exercícios dinâmicos (caminhada, ciclismo, ginástica, natação, entre outras), todos os dias da semana, com duração mínima de 30 minutos. Moderadamente ativos os usuários que relataram realizar atividades físicas com frequência de no mínimo três vezes por semana e com duração mínima de 30 minutos $^{(11)}$.

Foram classificados como etilistas os usuários que relataram fazer consumo ativo de bebidas alcoólicas diariamente, por no mínimo duas vezes ao dia. Esta variável foi verificada de acordo com o diagnóstico médico e anotações de acompanhamento com o profissional psicólogo, disponível nos prontuários e que tiveram diagnóstico classificado na Classificação Internacional de Doenças (CID-10), com a classificação F10 (Transtornos mentais e de comportamento decorrentes do uso de álcool) ${ }^{(16)}$. Para as variáveis referentes às orientações, efeitos colaterais da medicação em uso e dieta nutricional prescrita por profissional de saúde, foram consideradas as respostas referidas pelos depoentes e informações contidas nos prontuários.

Todos os questionários foram checados, tabulados em planilha eletrônica e posteriormente analisados por meio do programa IBM SPSS versão 20.0. Utilizou-se o modelo de regressão logística, com o método stepwise Forward, considerando todas as variáveis que apresentaram o p-valor $<0,20$ na análise univariada e se mantiveram no modelo multivariado apenas as variáveis que resultaram o valor de $p<0,05$. A magnitude das associações foi estimada pelo cálculo de Odds Ratio (OR), adotando o intervalo de confiança de $95 \%$ como medida de precisão.

A pesquisa foi apreciada pelo Comitê Permanente de Ética em Pesquisa com Seres Humanos, recebendo parecer favorável (1.407.687/2016). 
Foram analisadas 417 pessoas, entre estas, 260 (62,4\%) com idade $\geq 60$ anos, 283 (67,8\%) do sexo feminino, 260 (62,3\%) da cor branca, $183(43,8 \%)$ pertenciam à classificação econômica de extrato C, $255(61,1 \%)$ com ensino fundamental, 230 (55,2\%) eram aposentados e/ou pensionistas e 249 (59,6\%) moravam com companheiro e filhos (Tabela 1).

Tabela 1 - Análise univariada dos fatores associados à pressão arterial inadequada de pessoas com hipertensão acompanhadas pela Estratégia Saúde da Família, segundo perfil sociodemográfico. Maringá, PR, Brasil, 2016

\begin{tabular}{|c|c|c|c|c|c|}
\hline & \multicolumn{2}{|c|}{ Pressão Arterial } & \multicolumn{3}{|c|}{ Análise Univariada } \\
\hline & Adequada + & Inadequada + & OR & IC 95\% & $\mathbf{p}$ \\
\hline \multicolumn{6}{|l|}{ Idade } \\
\hline 20 a 59 anos & $95(22,8)$ & $62(14,8)$ & 1 & & \\
\hline$>60$ anos & $129(30,9)$ & $131(31,5)$ & 1,55 & $1,04-2,32$ & $0,031^{*}$ \\
\hline \multicolumn{6}{|l|}{ Sexo } \\
\hline Masculino & $74(17,8)$ & $60(14,4)$ & 1 & & \\
\hline Feminino & $150(35,9)$ & $133(31,9)$ & 1,09 & $0,72-1,65$ & 0,671 \\
\hline \multicolumn{6}{|l|}{ Situação Familiar } \\
\hline Mora com Companheiro e Filho & $141(33,7)$ & $108(25,9)$ & 1 & & \\
\hline Mora sem Companheiro e Filho & $50(12,0)$ & $45(10,8)$ & 1,33 & $0,83-2,14$ & 0,234 \\
\hline Vive Só & $33(8,0)$ & $40(9,6)$ & 1,34 & $0,79-2,26$ & 0,27 \\
\hline \multicolumn{6}{|l|}{ Escolaridade } \\
\hline Não sabe ler/escrever & $20(4,8)$ & $12(2,9)$ & 1,52 & $0,51-4,53$ & 0,445 \\
\hline Ensino Fundamental & $131(31,4)$ & $124(29,7)$ & 1,57 & $0,74-3,36$ & 0,238 \\
\hline Ensino Médio & $61(14,6)$ & $46(11,1)$ & 1,25 & $0,55-2,83$ & 0,581 \\
\hline Ensino Superior & $12(2,9)$ & $11(2,6)$ & 1 & & \\
\hline \multicolumn{6}{|l|}{ Cor } \\
\hline Branco & $138(33,1)$ & $122(29,2)$ & 1 & & \\
\hline Preto & $37(8,8)$ & $28(6,7)$ & 0,85 & $0,49-1,48$ & 0,578 \\
\hline Pardo & $49(11,8)$ & $43(10,3)$ & 0,99 & $0,61-1,59$ & 0,976 \\
\hline \multicolumn{6}{|l|}{ Ocupação } \\
\hline Empregado & $58(13,9)$ & $38(9,1)$ & 1 & & \\
\hline Desempregado & $56(13,4)$ & $35(8,4)$ & 0,95 & $0,53-1,71$ & 0,875 \\
\hline Aposentado & $110(26,4)$ & $120(28,8)$ & 1,66 & $1,02-2,70$ & $0,039 *$ \\
\hline \multicolumn{6}{|l|}{ Classificação Econômica - ABEP } \\
\hline $\mathrm{A}$ e $\mathrm{B}$ & $75(18,0)$ & $73(17,6)$ & 1 & & \\
\hline $\mathrm{C}$ & $98(23,5)$ & $85(20,3)$ & 0,89 & $0,57-1,37$ & 0,891 \\
\hline De E & $51(12,2)$ & $35(8,4)$ & 0,7 & $0,41-1,20$ & 0,203 \\
\hline
\end{tabular}

Legenda: †: n (\%); OR: Odds Ratio; IC: índice de Confiança; *valor de $\mathrm{p}<0,20$, variáveis inclusas no modelo ajustado de regressão logística.

$\mathrm{Na}$ análise univariada, os resultados apresentaram que 224 (53,7\%) dos participantes do estudo mantinham a pressão arterial adequada e $262(62,8 \%)$ tinham o acompanhamento adequado na ESF. Já para as variáveis antropométricas, 166 (40,3\%) entrevistados apresentaram sobrepeso, 287 (68,8\%) com circunferência abdominal alterada, 200 (48\%) não praticavam atividades físicas e $272(65,2 \%)$ utilizavam de um a dois medicamentos para tratamento da HA (Tabela 2). 
Cogitare Enferm. (23)1: e54087, 2018

Tabela 2 - Análise univariada dos fatores associados à pressão arterial inadequada, segundo perfil clínico e antropométrico. Maringá, PR, Brasil, 2016

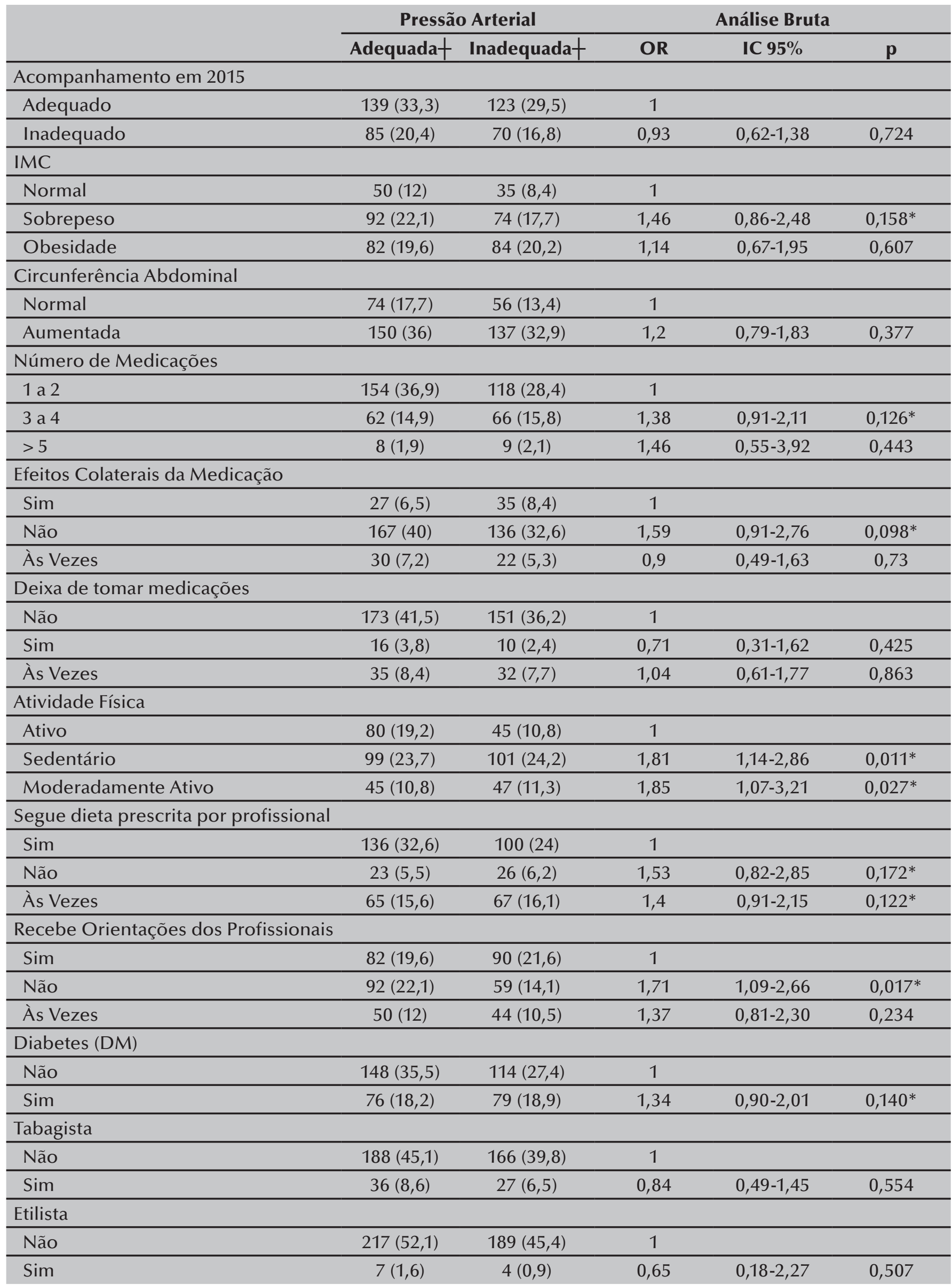

Legenda: +: n (\%); OR: Odds Ratio; IC: índice de Confiança; *valor de $\mathrm{p}<0,20$, variáveis inclusas no modelo multivariado de regressão logística. 
Dos entrevistados, $236(56,6 \%)$ relataram que seguiam dieta nutricional prescrita por profissionais de saúde e 172 (41,2\%) disseram receber informações sobre a morbidade, tratamento e uso adequado das medicações, 155 (37,2\%) eram pessoas com DM, 63 (15,1\%) eram tabagistas ativos e 11 (2,6\%) faziam consumo diário de bebida alcoólica (Tabela 2).

$\mathrm{Na}$ análise ajustada, os resultados apontam que as pessoas com idade $\geq 60$ anos e aposentados/ pensionistas, possuem chances de 1,47 vezes $($ IC $95 \%=1,27-2,04)$ e chances de 1,53 vezes (IC95\% $=1,13$ 2,18), respectivamente, de apresentar controle pressórico inadequado (Tabela 3 ).

Tabela 3 - Análise multivariada dos fatores associados à pressão arterial inadequada. Maringá, PR, Brasil, 2016

\begin{tabular}{lccccc} 
& \multicolumn{2}{c}{ Pressão Arterial } & Análise Ajustada \\
\hline & Adequada & Inadequada & ORaj & IC 95\% & p \\
\hline Idade & \multicolumn{5}{c}{1} \\
\hline 20 a 59 anos & $95(22,8)$ & $62(14,8)$ & & \\
\hline$>60$ anos & $129(30,9)$ & $131(31,5)$ & 1,47 & $1,27-2,04$ & 0,018 \\
\hline Ocupação & & & & \\
\hline Empregado & $58(13,9)$ & $38(9,1)$ & 1 & & \\
\hline Desempregado & $56(13,4)$ & $35(8,4)$ & 0,89 & $0,49-1,63$ & 0,725 \\
\hline Aposentado & $110(26,4)$ & $120(28,8)$ & 1,53 & $1,13-2,18$ & 0,034 \\
\hline Atividade Física & & & & \\
\hline Ativo & $80(19,2)$ & $45(10,8)$ & 1 & & \\
\hline Sedentário & $99(23,7)$ & $101(24,2)$ & 1,83 & $1,11-2,99$ & 0,016 \\
\hline Moderadamente Ativo & $45(10,8)$ & $47(11,3)$ & 1,94 & $1,09-3,46$ & 0,024 \\
\hline Recebe Orientações dos Profissionais & & & & \\
\hline Sim & $82(19,6)$ & $90(21,6)$ & 1 & & \\
\hline Não & $92(22,1)$ & $59(14,1)$ & 1,69 & $1,06-2,69$ & 0,025 \\
\hline Às Vezes & $50(12)$ & $44(10,5)$ & 1,26 & $0,73-2,18$ & 0,395
\end{tabular}

Legenda: †: n (\%); OR: OddsRatio; IC: índice de Confiança. Permaneceram no modelo apenas as variáveis que apresentaram valor de $p<0,05$.

As pessoas que relataram não praticar atividade física e as que são moderadamente ativas possuem chances de 1,83 vezes (IC95\%=1,11-2,99) e 1,94 chances (IC95\%=1,09-3,46), respectivamente, de pressão arterial inadequada. Os que referiram não receber nenhum tipo de informações sobre as complicações da HA, incentivo às práticas de autocuidado, uso correto das medicações e de possíveis efeitos colaterais, possuem chance de 1,69 vezes $($ IC95\% $=1,06-2,69)$ de possuir a pressão arterial inadequada (Tabela 3).

\section{- DISCUSSÃO}

Este estudo identificou elevada ocorrência (46,7\%) de pessoas em tratamento da HA, acompanhadas

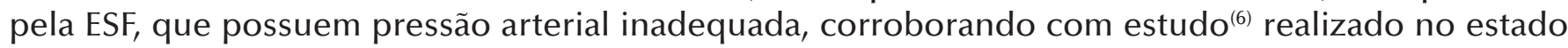
do Paraná, com população semelhante e que mostrou a não adesão à terapia medicamentosa como principal fator de risco para inadequação dos valores pressóricos. Estudo realizado na Indonésia ${ }^{(2)}$ apontou que apenas $25 \%$ dos entrevistados tinham o controle pressórico adequado, evidenciando como determinantes a fragilidade no diagnóstico prévio e fatores relacionados ao conhecimento sobre a doença.

Neste estudo, evidenciou-se maior prevalência de mulheres com hipertensão, escolaridade e classificação econômica baixa e que convivem com familiares, corroborando com estudo realizado em 
São Paulo(3), justificando as mulheres como mais atuantes na busca pelo diagnóstico e tratamento de doenças crônicas, e aderindo mais ao tratamento.

Identificou-se associação significativa entre a pressão arterial inadequada e as pessoas com idade superior a 59 anos e os aposentados/pensionistas. Resultados semelhantes foram encontrados em estudo realizado no Ceará(5). Pode-se inferir a importância da atenção voltada à pessoa da terceira idade e como a situação econômica intervém no processo de saúde doença. A idade avançada e o poder aquisitivo menor podem implicar no conhecimento sobre a doença e na compreensão sobre as medicações, orientações de autocuidado, mudança nos hábitos de vida e alimentares, contribuindo para a pressão arterial inadequada ${ }^{(3)}$.

Apesar de não apontar associação significativa dos marcadores antropométricos e a pressão arterial inadequada, a alta prevalência de pessoas com sobrepeso e obesidade identificada no estudo é preocupante, e apresentou dados semelhantes a estudo realizado na Indonésia ${ }^{(2)}$, que aponta crescimento da população obesa, que causa efeitos deletérios à saúde e interfere diretamente no sucesso do tratamento.

Na análise multivariada, o IMC não apresentou significância estatística com a pressão arterial inadequada, mas é preocupante o número de pessoas com sobrepeso $(39,8 \%)$, como também, os valores da circunferência abdominal alterada $(68,9 \%)$, corroborando com outros estudos realizados no país ${ }^{(17-18)}$. Nestes aspectos, aponta-se a necessidade de estratégias promocionais para redução de peso e mudanças nos hábitos alimentares, considerando que a adiposidade possui potencial efeito no mecanismo fisiológico adequado do organismo, contribuindo para aumento da pressão arterial ${ }^{(17)}$.

O Ministério da Saúde (MS) e a SBC sugerem a adoção de ações que incentivem a prática de autocuidado, com uma abordagem cognitiva e comportamental do usuário, com propósito de avaliar o seu consumo alimentar e de que maneira as mudanças podem ocorrer sem causar impacto na dinâmica social, econômica, familiar e religiosa dessas pessoas ${ }^{(19)}$. No caso de pessoas com HA, o incentivo à dieta hipossódica é uma medida que pode assegurar o controle pressórico, uma vez que o consumo em excesso de sódio é associado a sintomas tensionais da doença ${ }^{(11,20)}$.

Não obstante, a maioria dos entrevistados relatou que segue dieta nutricional prescrita por profissionais de saúde. Estudo realizado em Porto Alegre, que avaliou o conhecimento das pessoas com HA sobre os alimentos ricos em sódio, resultou em um conhecimento insuficiente, considerando que os sujeitos possuem acompanhamento regular em ambulatório especializado, apontando a necessidade de orientações nutricionais e incentivo aos hábitos alimentares saudáveis ${ }^{(21)}$.

Nesse aspecto, as ações assistenciais providas pela equipe de enfermagem pelos profissionais de nutrição, que atuam no Núcleo de Apoio à Saúde da Família (NASF), com ações de avaliação de uma dieta adequada e prescrição de um plano dietético, de caráter individual e efetivo à população assistida por sua equipe e que também é sugerida pelo MS. Estas ações necessitam ser definidas a partir da gestão de caso, com a equipe médica responsável pelo acompanhamento e avaliação dos resultados ${ }^{(20,22)}$.

Outra medida a ser adotada pelos profissionais de saúde é o incentivo à realização de atividades físicas, também recomendadas pelo $\mathrm{MS}$ e pela $\mathrm{SBC}^{(11,19-20)}$, como prática para regulação do peso corpóreo, redução do acúmulo da adiposidade abdominal e melhora dos sintomas tensionais da HA. Neste estudo, a prática de atividade física apresentou associação significativa com a pressão arterial inadequada, em que as pessoas sedentárias e que fazem atividades físicas com pouca frequência são mais vulneráveis a apresentar dificuldade em manter a pressão arterial adequada, assemelhando-se a um ensaio clínico randomizado, realizado em uma população com características clínicas semelhantes ${ }^{(23)}$.

Da mesma forma, os dados corroboram com estudo realizado no interior de São Paulo, em que a maioria dos entrevistados que relatou praticar atividade física apresentou adequação dos valores pressóricos. A prática orientada de atividades físicas auxilia no controle pressórico devido a sua ação hipotensora sobre o organismo, além de contribuir na redução e manutenção do peso corpóreo. Contudo, sua indicação necessita ser equitativa, de acordo com a condição clínica, limitações físicas e de saúde ${ }^{(11,17,20)}$.

Reitera-se que apesar dos resultados não apontarem associação da pressão arterial inadequada com 
o número de medicamentos em uso, é necessário que as orientações quanto ao uso das medicações e possíveis efeitos colaterais sejam realizadas frequentemente pelos profissionais de saúde ${ }^{(24-25)}$. Em estudo realizado com população de características clínicas semelhantes à presente pesquisa, os resultados apontaram o conhecimento insuficiente sobre a doença, sintomas e tratamento e a complexidade das medicações prescritas, como fatores de risco para não adesão à terapia farmacológica ${ }^{(24)}$.

Estudo $^{(25)}$ realizado em Marília-SP identificou que os entrevistados, mesmo classificados como não aderentes, se negavam a relatar que não faziam a adesão à terapia farmacológica e apontavam a importância de seguir o plano terapêutico, sendo esta contradição justificada pelos fatores sociais e culturais dos participantes do estudo. Os autores concluíram que vários motivos influenciaram em não adesão, como acontecimento de algo diferente na rotina diária, esquecer-se de tomar medicações e também o próprio conhecimento sobre a medicação, o que denota a importância das orientações e questionamento dos profissionais sobre adoção do tratamento contínuo e assíduo, conforme prescrito pela equipe médica e de encaminhamento a consulta com o profissional psicólogo para que se tenha vínculo e garantia da adesão à terapêutica de escolha.

Não obstante, as orientações prestadas pelos profissionais foram relatadas como insuficientes e apresentaram associação significativa entre aqueles com pressão arterial inadequada. Os profissionais de saúde necessitam atender necessidades de saúde da população, principalmente os que exercem sua função na APS, para que a adesão ao tratamento seja efetiva e os índices de mortalidade e de internações provenientes da cronicidade da doença sejam evitados, estimulando a autonomia e melhora na qualidade de vida da pessoa em condição crônica. Cabe também ao Estado o dever de cumprir suas obrigações quanto ao investimento de recursos na área da saúde para que a assistência seja realizada ${ }^{(6)}$.

O estudo evidenciou ainda um número baixo de pessoas que fazem consumo diário de álcool, assemelhando-se a estudo ${ }^{(5)}$ realizado com idosos portadores de hipertensão, residentes em um município do estado do Ceará, justificando-o um fator de risco modificável que pode ser solucionado com ações de educação em saúde. Considera-se o consumo abusivo de álcool como fator que dificulta a adesão ao tratamento farmacológico, principalmente pelos efeitos deletérios provocados pela sua ingestão e também sobre as crenças culturais e populares das pessoas sobre a interação da bebida com a medicação ${ }^{(15)}$.

Apesar dos resultados apresentarem um número expressivo de pessoas com HA e que possuem a DM como morbidade associada, não se associou significativamente em relação à pressão arterial inadequada, o que corrobora com estudo ${ }^{(5)}$ realizado no Ceará. No entanto, reitera-se a importância de avaliar a carga do diabetes na qualidade de vida de pessoas com HA, principalmente pelo seu potencial limitante de atribuições diárias. De acordo com o grau de cronicidade, o diabetes pode acarretar vários problemas relacionados às suas complicações, como cegueira, amputação de membros e agravamento cardiovascular, sendo uma doença preocupante e desafiadora para as políticas públicas de saúde no Brasil e no mundo, necessitando que os profissionais de saúde estejam instrumentalizados para utilizar medidas efetivas no controle e prevenção de agravos relacionados à morbidade ${ }^{(4-5,20)}$.

O estudo limita-se ao instrumento que aborda variáveis de forma autorreferida, como a prática de atividades físicas. Há diversos instrumentos na literatura que discutem de forma mais abrangente o tema, principalmente os estudos recomendados pelo Ministério da Saúde, como o VIGITEL ${ }^{(18)}$. O viés de casualidade reversa, que é inerente a estudos com essa abordagem metodológica, pode ter alterado resultados de algumas associações.

Por fim, sugere-se a realização de estudos com os profissionais para a compreensão das dificuldades em realizar as atividades diárias atribuídas a sua função e que comprometem a saúde das pessoas que dependem da oferta dos serviços públicos de saúde, contribuindo para novas estratégias de gestão organizacional, com oferta de serviços efetivos e resolutivos, pautados na prevenção de agravos e redução de custos com internações por causas evitáveis.

\section{CONCLUSÃO}


Constatou-se que as pessoas com idade superior a 59 anos e que são aposentadas/pensionistas apresentaram pressão arterial inadequada, sendo necessário um acompanhamento mais criterioso pelos profissionais de saúde, considerando que pessoas com idade avançada e poder aquisitivo menor possuem mais dificuldade em adesão ao tratamento, de realizar práticas de autocuidado e de realizar mudança nos hábitos de vida, que podem interferir no controle pressórico.

O sedentarismo e a obesidade foram prevalentes no estudo, necessitando de novas medidas intervencionistas e resolutivas por parte dos profissionais de saúde, atuantes no processo de diagnóstico e tratamento de doenças crônicas e acompanhamento de resultados. A ausência de orientações por parte dos profissionais de saúde às pessoas com hipertensão foi associada à pressão arterial inadequada, necessitando que o planejamento e a organização do processo de trabalho sejam realizados de acordo com a necessidade da população, visando à redução de agravos decorrente das complicações da HA e ao Estado em atender às necessidades de recursos humanos e financeiros para realização das práticas assistenciais nas unidades de saúde.

\section{REFERÊNCIAS}

1. World Health Organization (WHO). Health statistics and information systems: estimates for 2000-2012: causespecific mortality. [Internet] Geneva: WHO; 2015 [acesso em 29 jun 2015]. Disponível: http://www.who.int/ healthinfo/global_burden_disease/estimates/en/index1.html.

2. Hussain MA, Mamun AA, Reid C, Huxley RR. Prevalence, Awareness, treatment and control of hypertension in indonesian adults aged $\geq 40$ years: findings from the Indonesia family life survey (IFLS). PLoS ONE. [Internet] 2016;11(8) [acesso em 20 set 2016]. Disponível: https://doi.org/10.1371/journal.pone.0160922.

3. da Silva SSBE, de Oliveira SFSB, Pierin AMG. The control of hypertension in men and women: a comparative analysis. Rev. esc. enferm. USP. [Internet] 2016;50(1) [acesso em 20 set 2016]. Disponível: http://dx.doi.org/10.1590/ S0080-623420160000100007.

4. Malta DC, Bernal RTI, Andrade SSCA, da Silva MMA, Velasquez-Melendez G. Prevalência de fatores associados com hipertensão arterial autorreferida em adultos brasileiros. Rev. Saúde Publica. [Internet] 2017;51(Suppl 1) [acesso em 20 set 2016]. Disponível: http://dx.doi.org/10.1590/s1518-8787.2017051000006.

5. Morais PCA, Moreira RP, de Lima PA, Silva MGF, Ferreira JDF, Rouberte ESC. Blood pressure, heart diseases and lifestyles of elderly. Rev. Rene [Internet] 2015;16(5) [acesso em 20 set 2016]. Disponível: http://www.revistarene. ufc.br/revista/index.php/revista/article/view/2162.

6. Barreto MS, Matsuda LM, Marcon SS. Factors associated with inadequate blood pressure control in patients of primary care. Esc. Anna Nery. [Internet] 2016;20(1) [acesso em 26 set 2016]. Disponível: http://dx.doi. org/10.5935/1414-8145.20160016.

7. Ministério da Saúde (BR). Secretaria de Atenção à Saúde. Departamento de Atenção Básica. Coberturas do Saúde da Família. [Internet] Brasília: Ministério da Saúde; 2016 [acesso em 26 mai 2016]. Disponível: http://dab. saude.gov.br/portaldab/historico_cobertura_sf.php.

8. Paraná. Instituto Paranaense de Desenvolvimento Econômico e Social (IPARDES). Caderno Estatístico Município de Maringá. [Internet] Curitiba: IPARDES; 2016 [acesso em 16 set 2016]. Disponível: http://www.ipardes.gov.br/ cadernos/MontaCadPdf1.php?Municipio=87000\&btOk=ok.

9. Kamakura W, Mazzon JA. Socioeconomic stratification criteria and classification tools in Brazil. Rev. adm. empres. [Internet] 2016;56(1) [acesso em 20 ago 2017]. Disponível: http://dx.doi.org/10.1590/S0034-759020160106.

10. Paes NA, Silva CS, Figueiredo TMRM, Cardoso MAA, Lima JO. Satisfação dos usuários hipertensos com os serviços da rede de atenção primária no Brasil: um estudo de validação. Rev Panam Salud Publica. [Internet] 2014;36(2) [acesso em 20 jul 2015]. Disponível: http://www.scielosp.org/pdf/rpsp/v36n2/03.pdf.

11. Malachias MVB, Souza WKSB, Plavnik FL, Rodrigues CIS, Brandão AA, Neves MFT, et al. Sociedade Brasileira de Cardiologia. $7^{\mathrm{a}}$ Diretriz Brasileira de Hipertensão Arterial. Arq Bras Cardiol. [Internet] 2016;107(3) [acesso em 18 ago 2016]. Disponível: http://www.scielo.br/pdf/abc/v107n3s3/0066-782X-abc-107-03-s3-0067.pdf. 
12. Ministério da Saúde (BR). Secretaria de Políticas de Saúde. Plano de Reorganização da Atenção à Hipertensão Arterial e ao Diabetes Mellitus. Brasília: Ministério da Saúde; 2001.

13. Silva CS, Paes NA, de Figueiredo TMRM, Cardoso MAA, Silva ATMC, Araújo JSS. Blood pressure control and adherence/attachment in hypertensive users of primary healthcare. Rev. esc. enferm. USP. [Internet] 2013;47(3) [acesso em 11 set 2016]. Disponível: http://dx.doi.org/10.1590/S0080-623420130000300009.

14. Associação Brasileira para o Estudo da Obesidade e da Síndrome Metabólica. Diretrizes brasileiras de obesidade 2016 / ABESO - Associação Brasileira para o Estudo da Obesidade e da Síndrome Metabólica. 4 ${ }^{\mathrm{a}}$ ed. [Internet] São Paulo (SP); 2016 [acesso em 03 nov 2016] Disponível: http://www.abeso.org.br/uploads/ downloads/92/57fccc403e5da.pdf.

15. Ministério da Saúde (BR). Secretaria de Atenção à Saúde. Departamento de Atenção Básica. Estratégias para o cuidado da pessoa com doença crônica: o cuidado da pessoa tabagista. Brasília: Ministério da Saúde; 2015.

16.GigliottiaA, BessaMA.SíndromedeDependênciadoÁlcool:Critérios diagnósticos. Rev. Bras.Psiquiatr. [Internet] 2004;26(Suppl 1) [acesso em 14 jun 2016]. Disponível: http://dx.doi.org/10.1590/S1516-44462004000500004.

17. Turi BC, Codogno JS, Fernandes RA, Monteiro HL. Prática de atividade física, adiposidade corporal e hipertensão em usuários do Sistema Único de Saúde. Rev. bras. epidemiol. [Internet] 2014;17(4) [acesso em 15 set 2016]. Disponível: http://dx.doi.org/10.1590/1809-4503201400040011.

18. Muraro AP, dos Santos DF, Rodrigues PRM, Braga JU. Fatores associados à Hipertensão Arterial Sistêmica autorreferida segundo VIGITEL nas 26 capitais brasileiras e no Distrito Federal em 2008. Ciênc. saúde coletiva. [Internet] 2013;18(5) [acesso em 21 set 2017]. Disponível: http://dx.doi.org/10.1590/S1413-81232013000500024.

19. Ministério da Saúde (BR). Secretaria de Atenção à Saúde. Departamento de Atenção Básica. Estratégias para o cuidado da pessoa com doença crônica: obesidade. Brasília: Ministério da Saúde; 2014.

20. Ministério da Saúde (BR). Secretaria de Atenção à Saúde. Departamento de Atenção Básica. Estratégias para o cuidado da pessoa com doença crônica. Brasília: Ministério da Saúde; 2014.

21. Teixeira JF, Goulart MR, Busnello FM, Pellanda LC. Conhecimento e atitudes sobre alimentos ricos em sódio por pacientes hipertensos. Arq. Bras. Cardiol. [Internet] 2016;106(5) [acesso em 26 set 2016]. Disponível: http:// dx.doi.org/10.5935/abc.20160049.

22. Ministério da Saúde (BR). Secretaria de Atenção à Saúde. Departamento de Atenção Básica. Política Nacional de Atenção Básica. Brasília: Ministério da Saúde; 2012.

23. Radovanovic CAT, Bevilaqua CA, Molena-Fernandes CA, Marcon SS. Multi-professional intervention in adults with arterial hypertension: a randomized clinical trial. Rev. bras. enferm. [Internet] 2016;69(6) [acesso em 10 mai 2017]. Disponível: http://dx.doi.org/10.1590/0034-7167-2016-0320.

24. Barreto MS, Reiners AAO, Marcon SS. Conhecimento sobre hipertensão arterial e fatores associados a não adesão à farmacoterapia. Rev. Latino-Am. Enfermagem. [Internet] 2014;22(3) [acesso em 14 out 2016]. Disponível: http://dx.doi.org/10.1590/0104-1169.3447.2442.

25. Marin NS, dos Santos MF, Moro AS. Perception of hypertensive patients about their non-adherence to the use of medication. Rev. esc. enferm. USP [Internet] 2016;50(n.esp) [acesso em 14 nov 2016]. Disponível: http:// dx.doi.org/10.1590/S0080-623420160000300009. 